\title{
Impact of Chemical Features on Methane Adsorption by Porous Materials at Varying Pressures
}

Maryam Pardakhti ${ }^{1}$, Pariksheet Nanda ${ }^{2}$, Ranjan Srivastava ${ }^{1,3^{*}}$

${ }^{1}$ Department of Chemical and Biomolecular Engineering, University of Connecticut, Storrs, CT 06269, United States

${ }^{2}$ Department of Molecular and Cell Biology, University of Connecticut, Storrs, CT 06269, United States

${ }^{3}$ Department of Biomedical Engineering, University of Connecticut, Storrs, CT 06269, United States

*Corresponding Author: rs@uconn.edu

Supporting Information 
a)

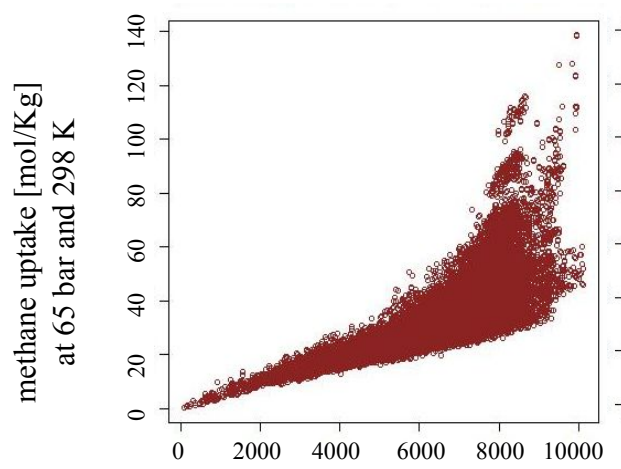

d)

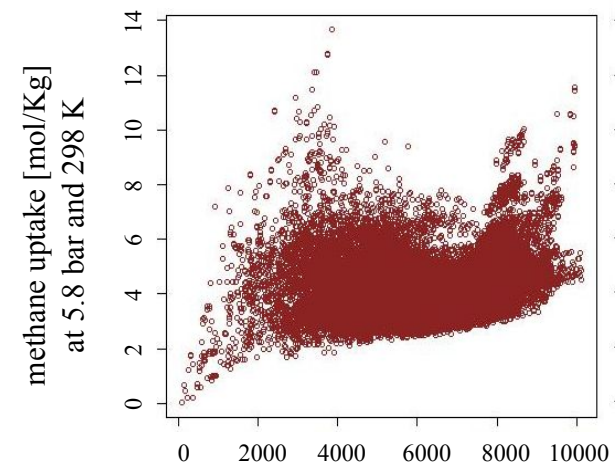

g)

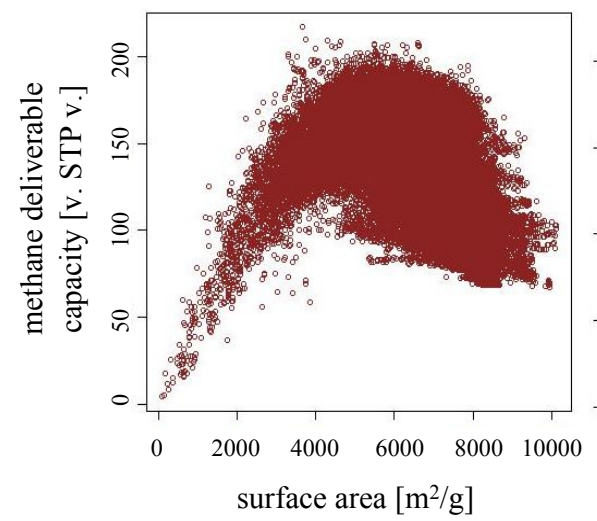

b)

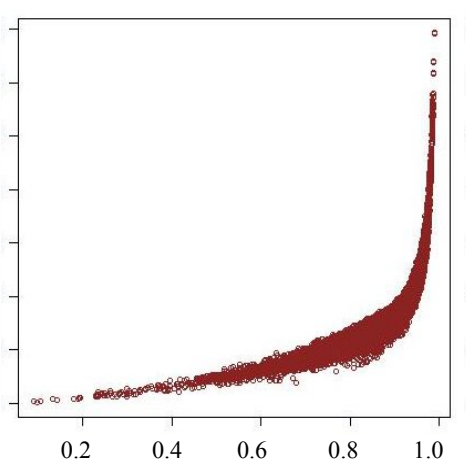

e)

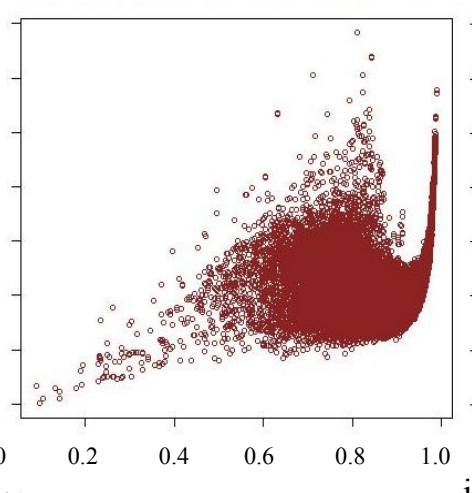

h)

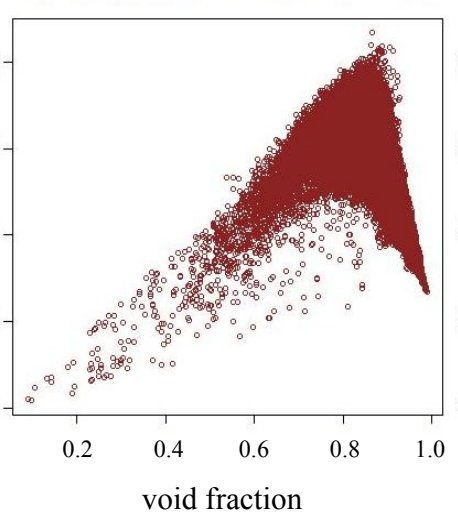

c)

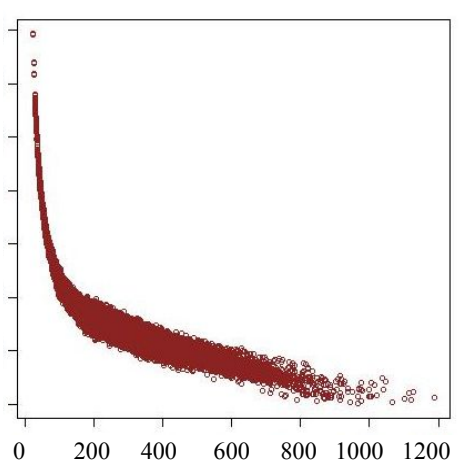

f)

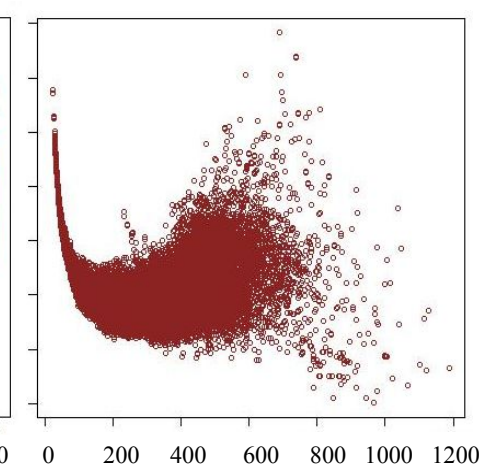
i)

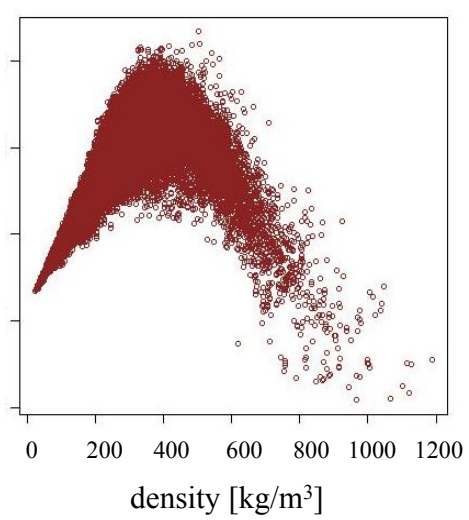

Figure S1. COFs methane adsorption vs. (a) surface area (b) void fraction (c) density at 65 bar and 298 K. Methane adsorption vs. (d) surface area (e) void fraction (f) density at 1 bar and $298 \mathrm{~K}$. Methane deliverable capacity vs. (g) surface area (h) void fraction (i) density. 
a)

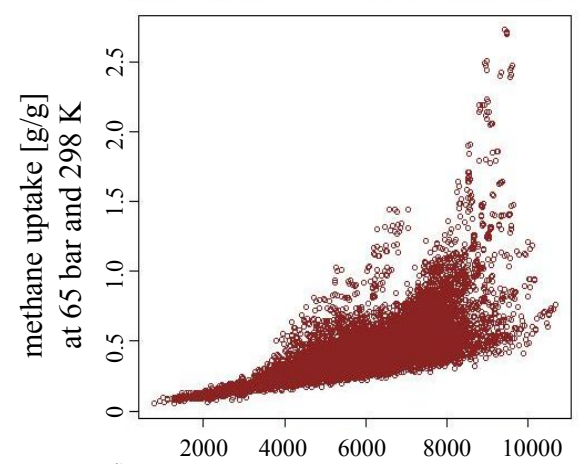

d)

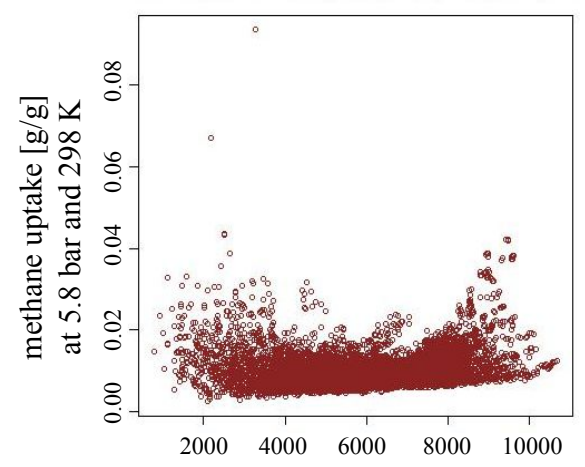

g)

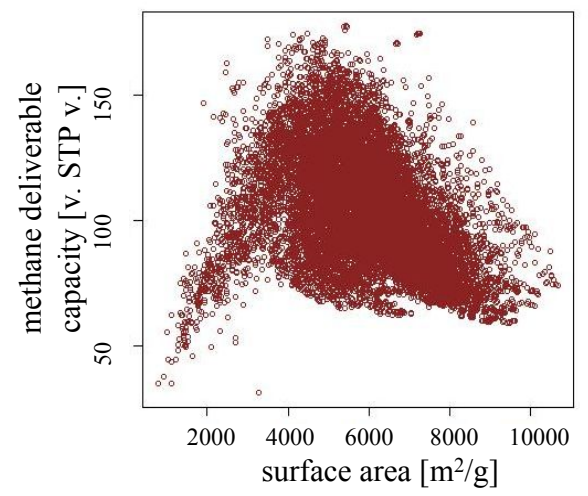

b)

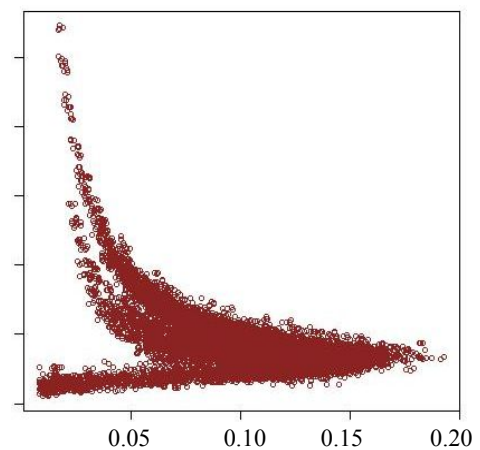

e)

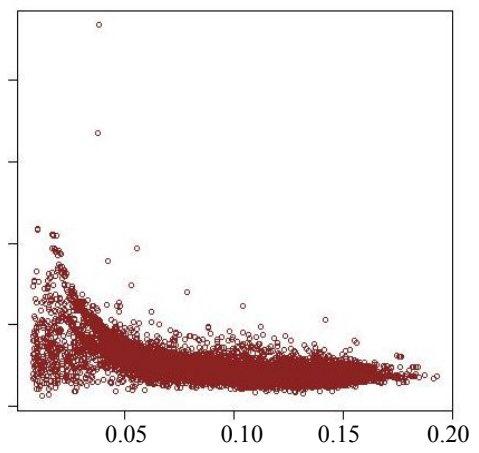

h)

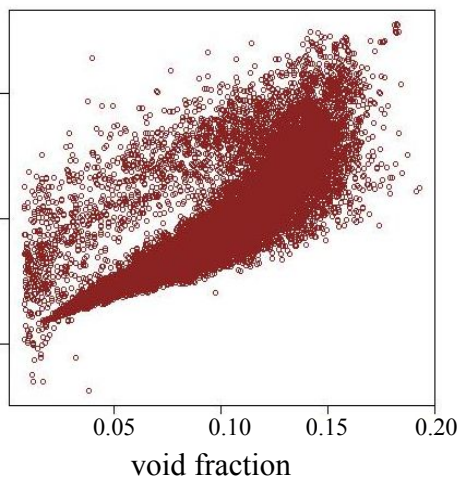

c)

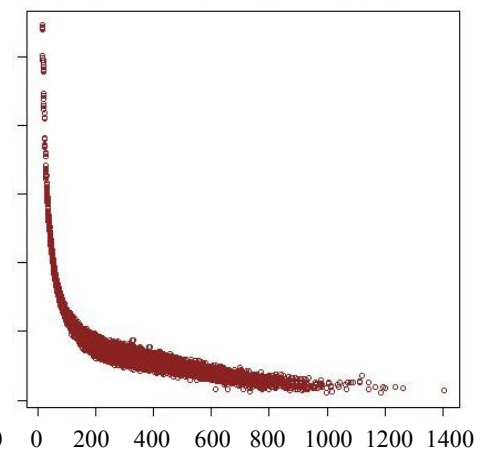

f)

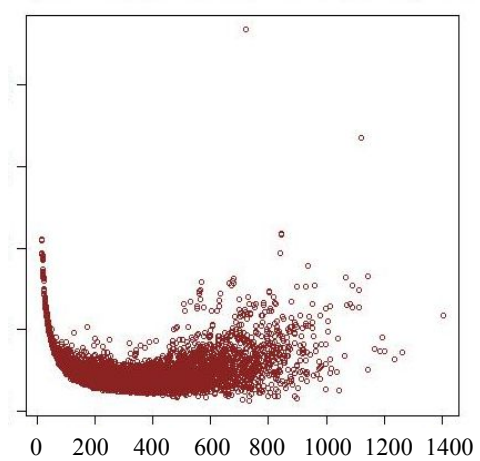

i)

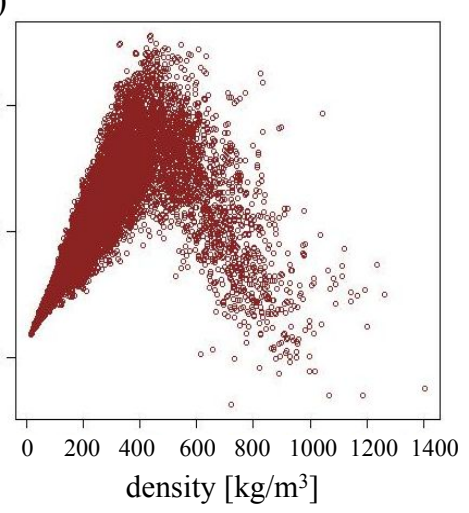

Figure S2. PPNs methane adsorption vs. (a) surface area (b) void fraction (c) density at 65 bar and $298 \mathrm{~K}$. Methane uptake vs. (d) surface area (e) void fraction (f) density on COFs at 5.8 bar and $298 \mathrm{~K}$. Methane deliverable capacity vs. (g) surface area (h) void fraction (i) density. 

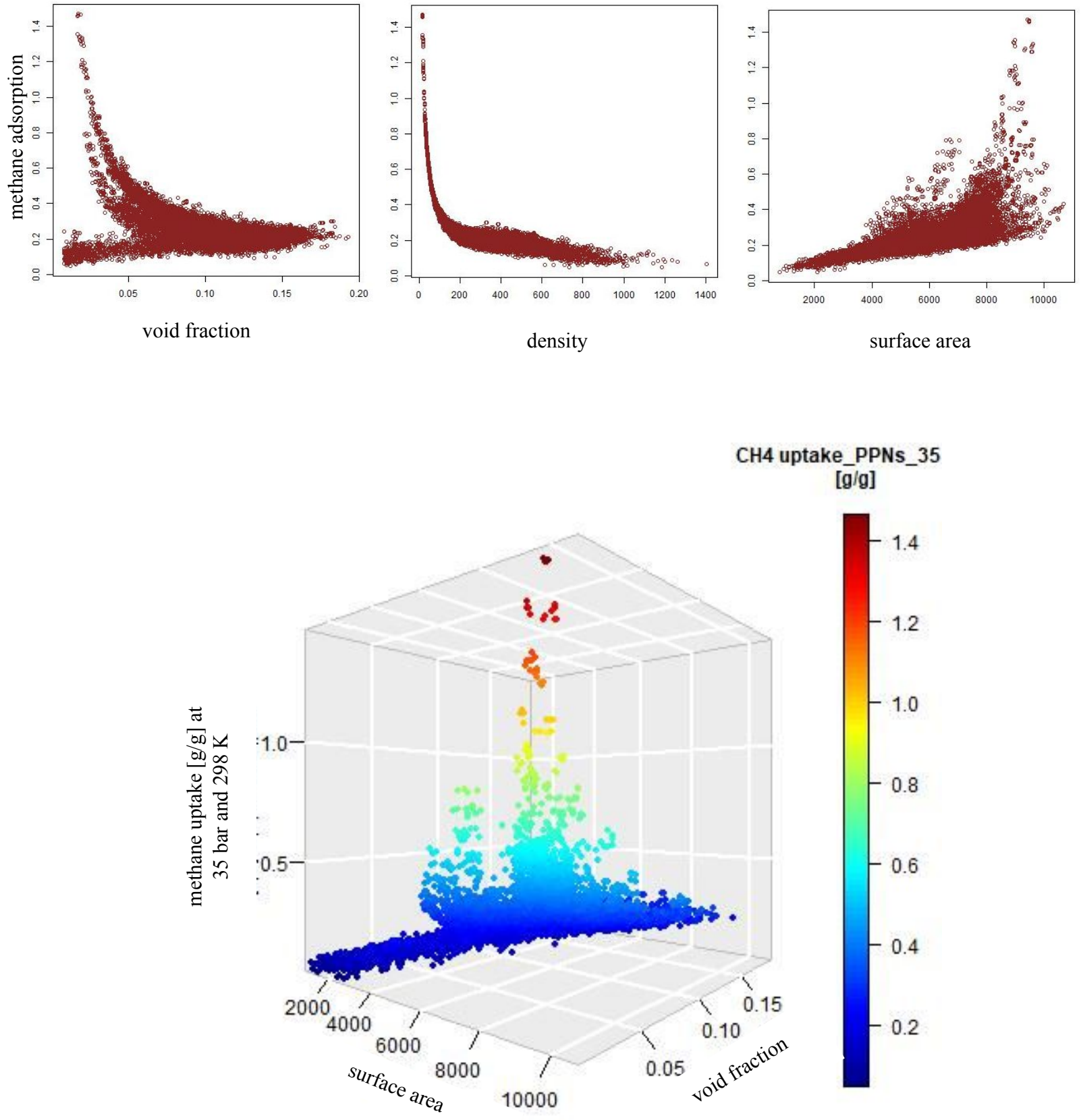

Figure S3(a). 3D presentation of methane uptake vs. surface area vs. void fraction on PPNs at 35 bar and $298 \mathrm{~K}$. 

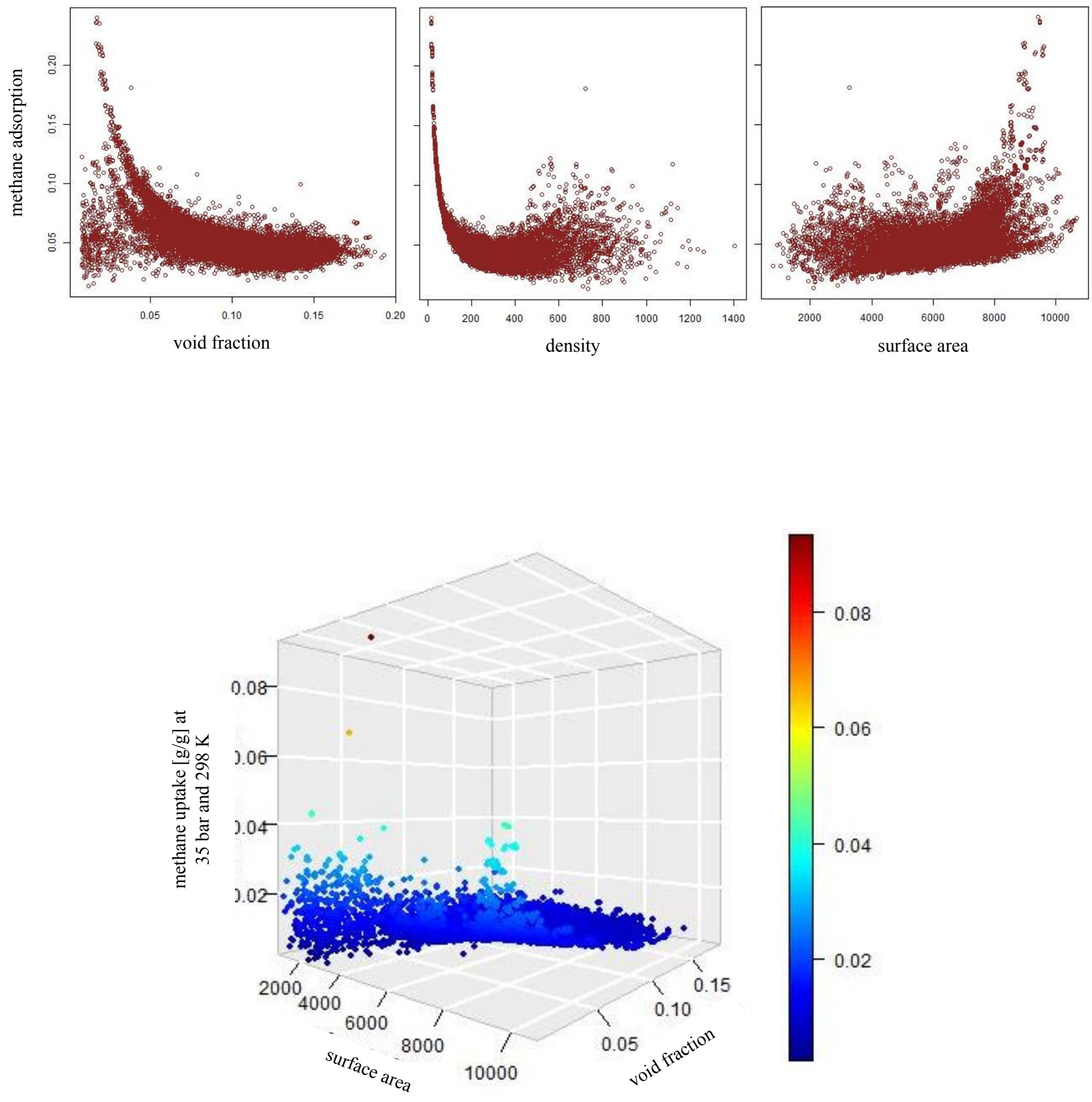

Figure S3(b). 3D presentation of methane uptake vs. surface area vs. void fraction on PPNs at 1 bar and $298 \mathrm{~K}$. 

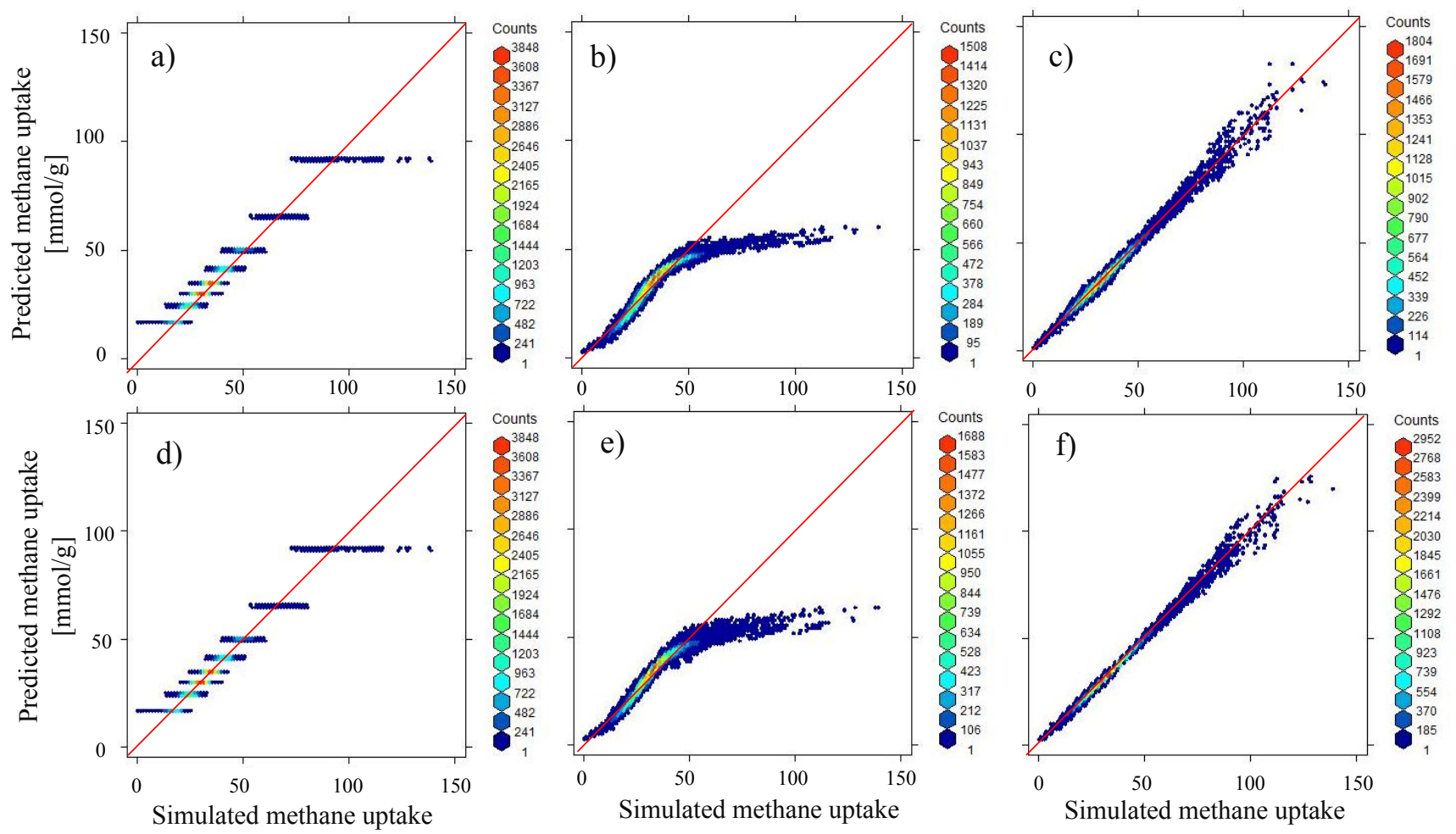

Figure S4. Methane adsorption [mmol/g] on COFs at 65 bar (a-c) using SO features, (d-f) using SC features. $(\mathrm{a}, \mathrm{d}) \mathrm{DT},(\mathrm{b}, \mathrm{e})$ Poisson regression, and $(\mathrm{c}, \mathrm{f}) \mathrm{RF}$ prediction algorithms.
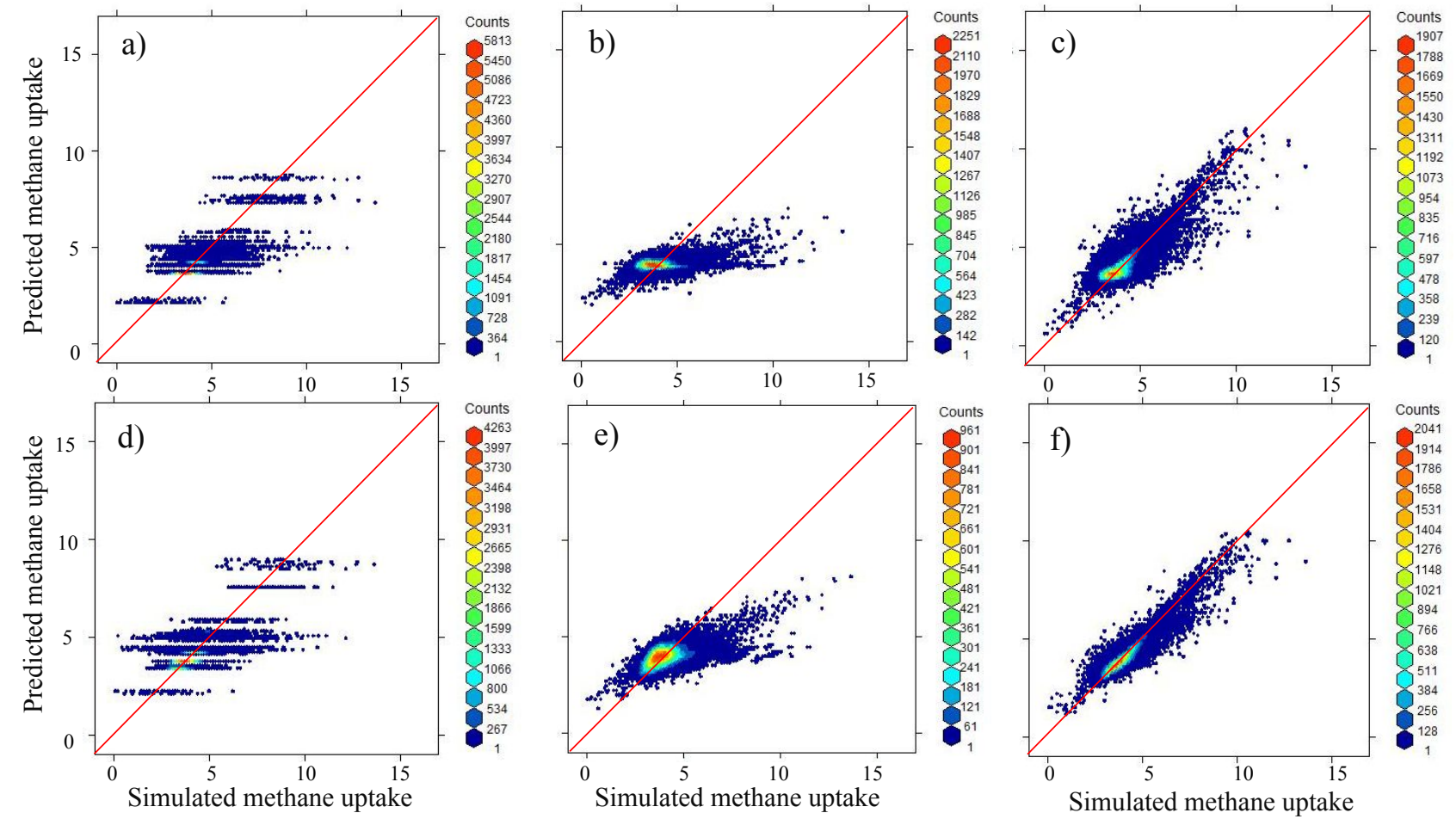

Figure S5. Methane adsorption [mmol/g] on COFs at 5.8 bar (a-c) using SO features, (d-f) using SC features. $(\mathrm{a}, \mathrm{d}) \mathrm{DT},(\mathrm{b}, \mathrm{e})$ Poisson regression, and $(\mathrm{c}, \mathrm{f}) \mathrm{RF}$ prediction algorithms 

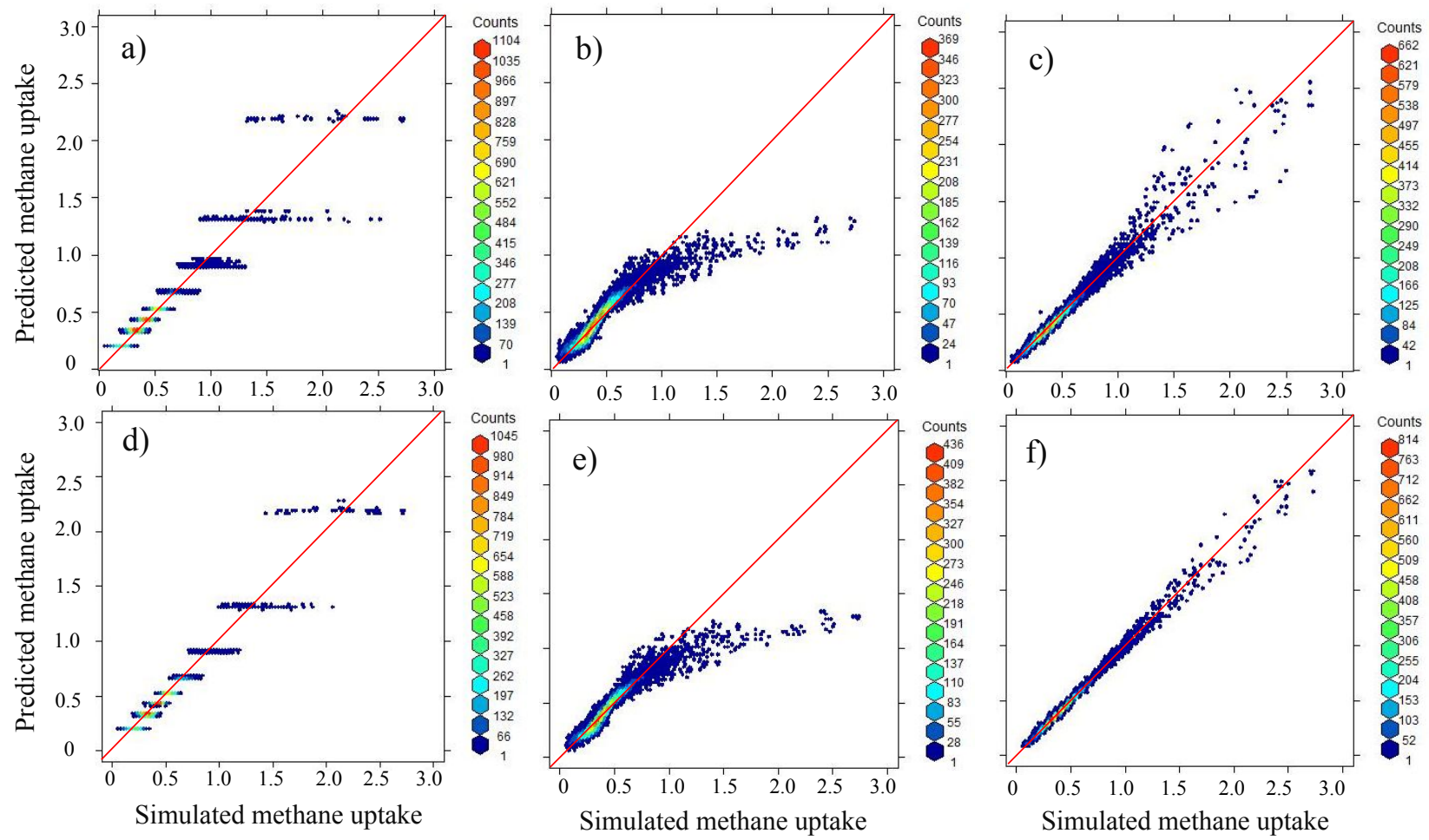

Figure S6. Methane adsorption [g/g] on PPNs at 65 bar (a-c) using SO features, (d-f) using SC features. $(\mathrm{a}, \mathrm{d})$ DT,$(\mathrm{b}, \mathrm{e})$ Poisson regression, and $(\mathrm{c}, \mathrm{f}) \mathrm{RF}$ prediction algorithms respectively.
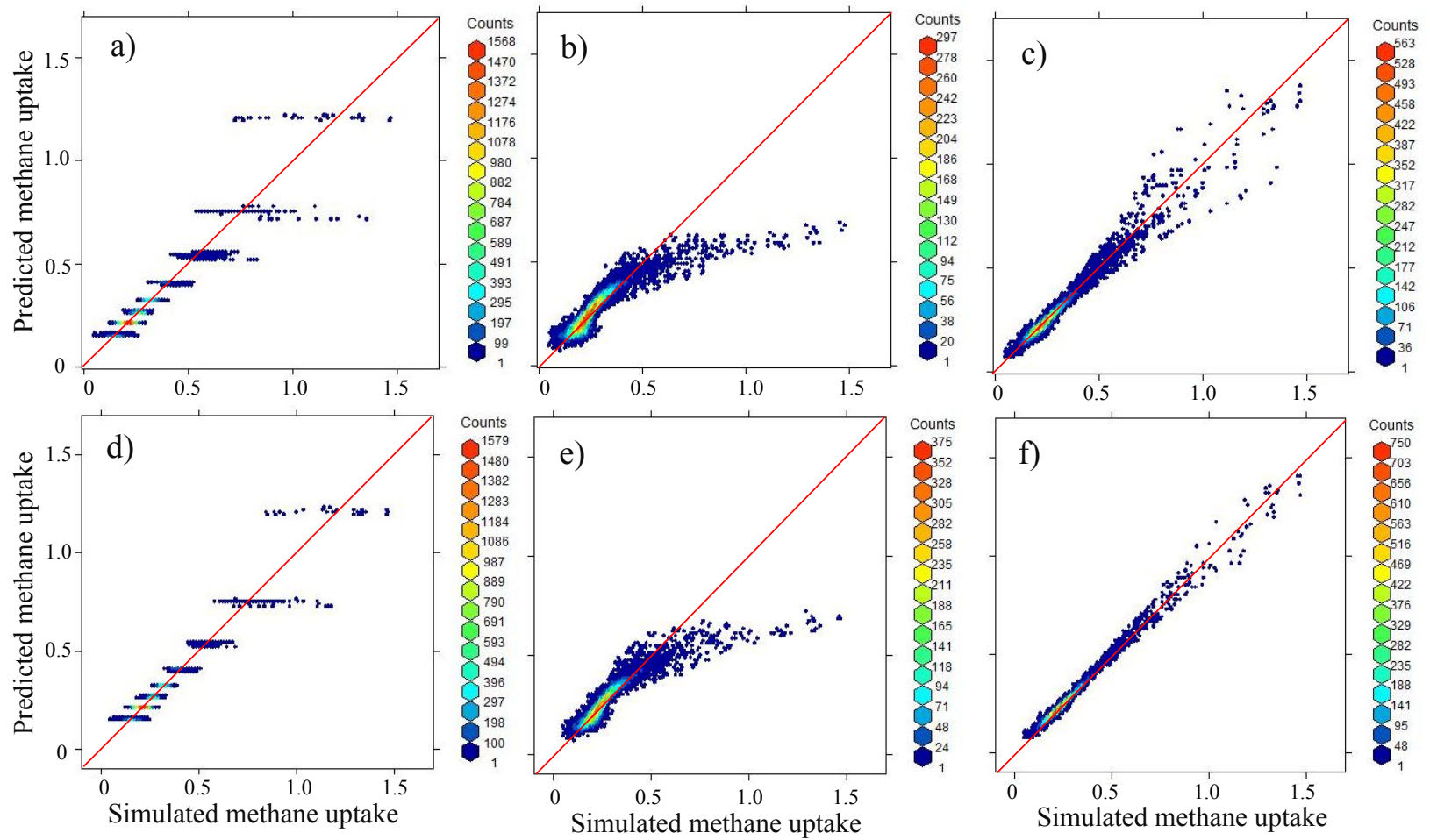

Figure S7. Methane adsorption [g/g] on PPNs at 35 bar (a-c) using SO features, (d-f) using SC features. $(\mathrm{a}, \mathrm{d}) \mathrm{DT},(\mathrm{b}, \mathrm{e})$ Poisson regression, and $(\mathrm{c}, \mathrm{f}) \mathrm{RF}$ prediction algorithms respectively. 

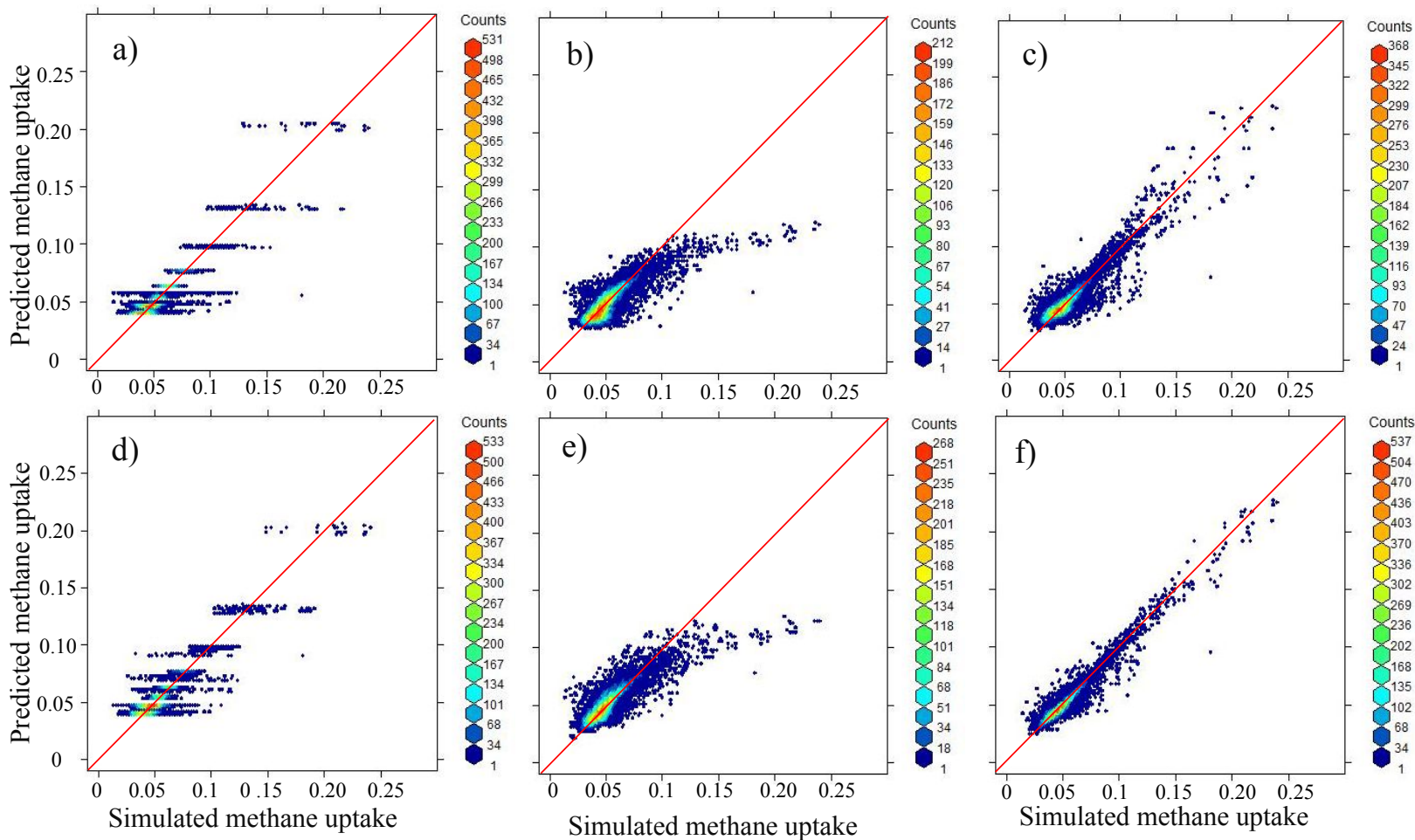

Figure S8. Methane adsorption $[\mathrm{g} / \mathrm{g}]$ on PPNs at 5.8 bar (a-c) using SO features, (d-f) using SC features. $(\mathrm{a}, \mathrm{d})$ DT,$(\mathrm{b}, \mathrm{e})$ Poisson regression, and $(\mathrm{c}, \mathrm{f}) \mathrm{RF}$ prediction algorithms respectively.
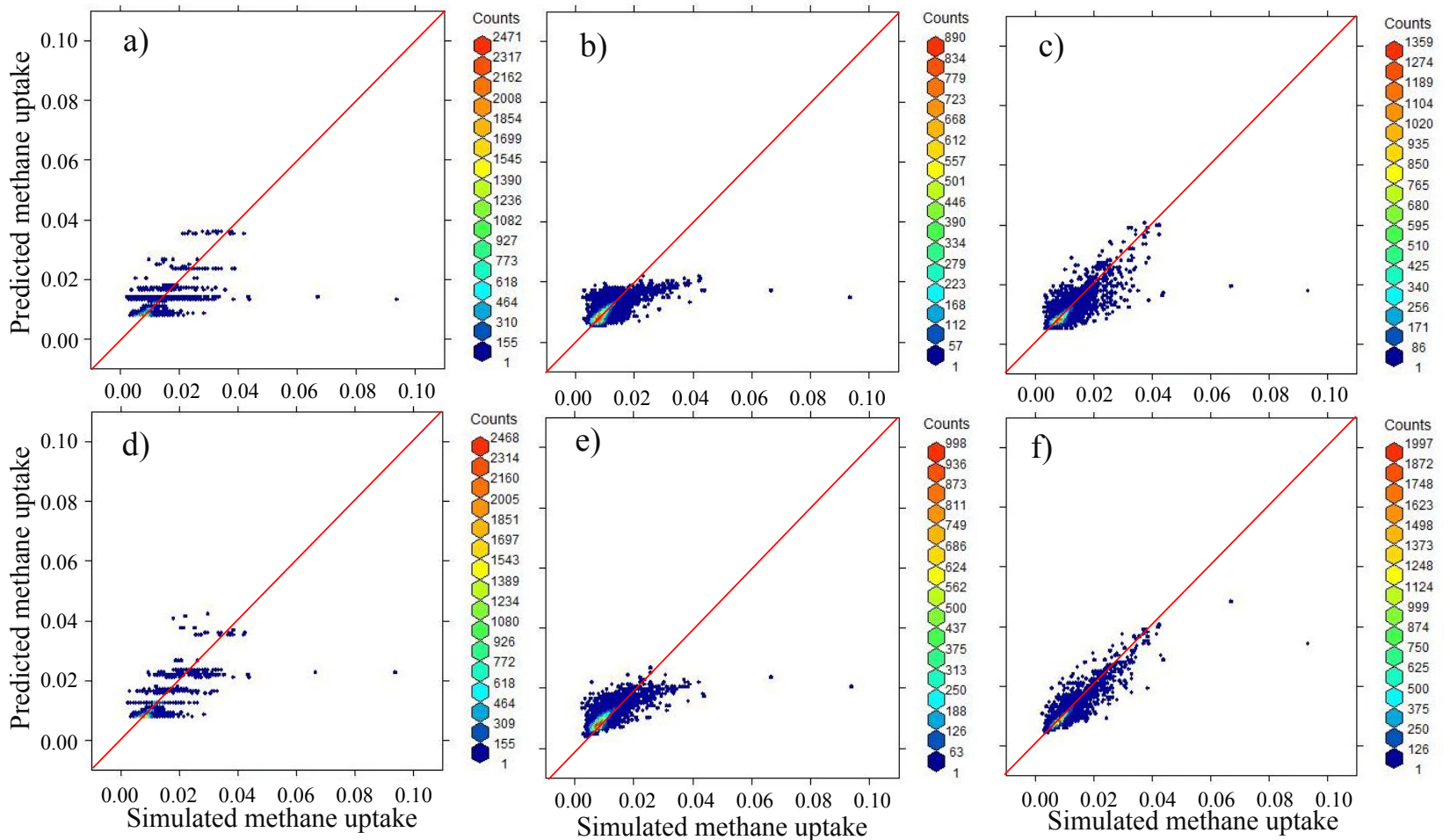

Figure S9. Methane adsorption $[\mathrm{g} / \mathrm{g}]$ on PPNs at 1 bar (a-c) using SO features, (d-f) using SC features. $(\mathrm{a}, \mathrm{d})$ DT , $(\mathrm{b}, \mathrm{e})$ Poisson regression, and $(\mathrm{c}, \mathrm{f}) \mathrm{RF}$ prediction algorithms respectively. 
Table S1. Prediction model performance using DT, Poisson, and RF models through SO and SC descriptors for COFs and PPNs.

\begin{tabular}{|c|c|c|c|c|c|c|c|c|}
\hline \multicolumn{9}{|c|}{ Prediction Model Performance } \\
\hline & \multirow{2}{*}{$\begin{array}{c}\text { Pressure } \\
\text { [bar] }\end{array}$} & \multirow{2}{*}{$\begin{array}{c}\text { Prediction } \\
\text { Model }\end{array}$} & \multicolumn{2}{|c|}{$\mathbf{R}^{2}$} & \multicolumn{2}{|c|}{ MAPE (\%) } & \multicolumn{2}{|c|}{$\begin{array}{c}\text { RMSE } \\
{[\mathrm{mol} / \mathrm{Kg}]}\end{array}$} \\
\hline & & & SO & SC & SO & SC & SO & SC \\
\hline \multirow{6}{*}{ COFs } & \multirow{3}{*}{65} & DT & 0.93 & 0.93 & 8.0 & 8.0 & 2.9 & 2.9 \\
\hline & & Poisson & 0.83 & 0.85 & 7.3 & 6.8 & 4.4 & 4.1 \\
\hline & & $\mathrm{RF}$ & 0.99 & 0.99 & 3.1 & 1.6 & 1.3 & 0.7 \\
\hline & \multirow{3}{*}{5.8} & DT & 0.51 & 0.53 & 10.3 & 9.8 & 0.56 & 0.54 \\
\hline & & Poisson & 0.06 & 0.27 & 13.7 & 11.8 & 0.77 & 0.68 \\
\hline & & $\mathrm{RF}$ & 0.66 & 0.87 & 8.7 & 5.1 & 0.46 & 0.30 \\
\hline \multirow{12}{*}{ PPNs } & \multirow{3}{*}{65} & DT & 0.90 & 0.93 & 11.1 & 10.9 & -3.75 & 3.75 \\
\hline & & Poisson & 0.85 & 0.86 & 10.4 & 9.7 & 5 & 5 \\
\hline & & $\mathrm{RF}$ & 0.97 & 0.99 & 4.99 & 3.3 & 2.5 & 1.25 \\
\hline & \multirow{3}{*}{35} & DT & 0.89 & 0.91 & 10.0 & 9.8 & 2.5 & 1.87 \\
\hline & & Poisson & 0.79 & 0.81 & 10.7 & 9.8 & 3.12 & 3.12 \\
\hline & & $\mathrm{RF}$ & 0.95 & 0.98 & 6.1 & 3.9 & 125 & 0.62 \\
\hline & \multirow{3}{*}{5.8} & DT & 0.75 & 0.78 & 11.7 & 11.4 & 0.62 & 0.5 \\
\hline & & Poisson & 0.67 & 0.72 & 12.1 & 10.7 & 0.62 & 0.56 \\
\hline & & $\mathrm{RF}$ & 0.83 & 0.93 & 9.7 & 6.0 & 0.62 & 0.25 \\
\hline & \multirow{3}{*}{1} & DT & 0.58 & 0.66 & 14.1 & 13.8 & 0.12 & 0.12 \\
\hline & & Poisson & 0.56 & 0.64 & 13.9 & 12.1 & 0.12 & 0.12 \\
\hline & & $\mathrm{RF}$ & 0.68 & 0.84 & 11.7 & 7.5 & 0.12 & 0.06 \\
\hline
\end{tabular}


a)

density

void fraction

weighted electronegativity

surface area

total degree of unsaturation

degree of unsaturation per carbon

number of carbon

number of fluorine

number of hydrogen

number of nitrogen

electronegative atoms to total atoms

number of sulfur

number of oxygen

number of silicon

nitrogen over oxygen ratio b)

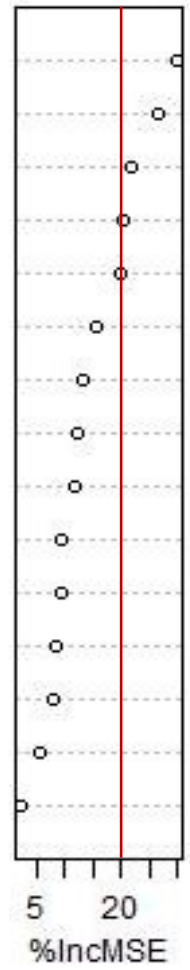

density

void fraction

number of nitrogen

surface area

degree of unsaturation per carbon

total degree of unsaturation

electronegative atoms to total atoms

weighted electronegativity

number of hydrogen

number of silicon

number of carbon

number of oxygen

nitrogen over oxygen ratio

number of sulfur

number of fluorine

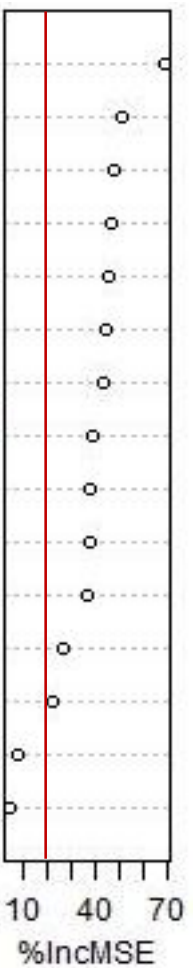

Figure S10. Feature importance (from random forest algorithm) for methane adsorption at high (65 bar) and low (5.8 bar) pressures on COFs. 
a)

density

total degree of unsaturation

electronegative atoms to total atoms

weighted electronegativity

number of hydrogen

void fraction

number of carbon

nitrogen over oxygen ratio

metal type Ge

surface area

number of oxygen

number of sulfur

number of nitrogen

number of germanium

number of fluorine

degree of unsaturation per carbon

number of silicon

metallic percentage

number of chlorine

number of iodine

number of phosphorus

b)

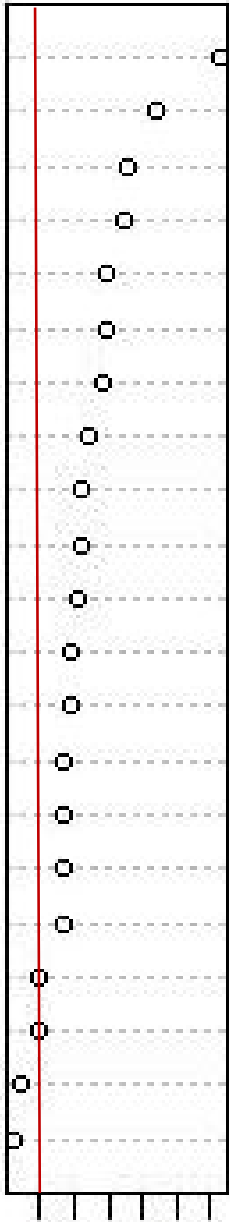

$10 \quad 40$ \%IncMSE density

void fraction

number of hydrogen

total degree of unsaturation

electronegative atoms to total atoms

surface area

number of carbon

degree of unsaturation per carbon

number of germanium

number of nitrogen

number of oxygen

nitrogen over oxygen ratio

metal type Ge

weighted electronegativity

number of silicon

metallic percentage

number of fluorine

number of sulfur

number of chlorine

number of phosphorus

number of iodine

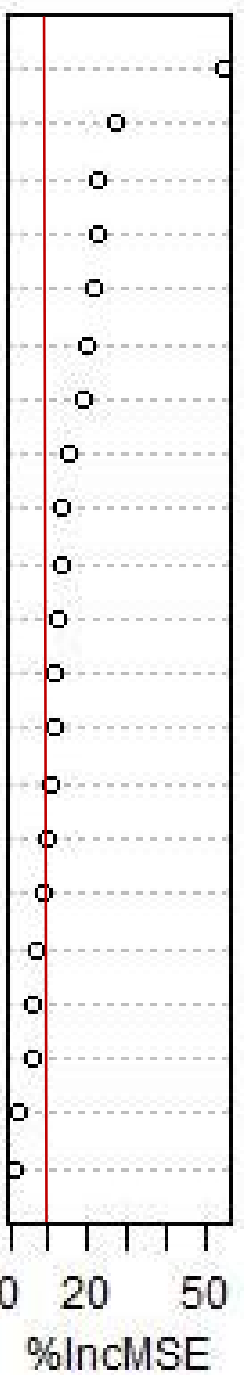

Figure S11. Feature importance (from random forest algorithm) for methane adsorption at high (65 bar) and low pressure (5.8 bar) on PPNs. 
a)

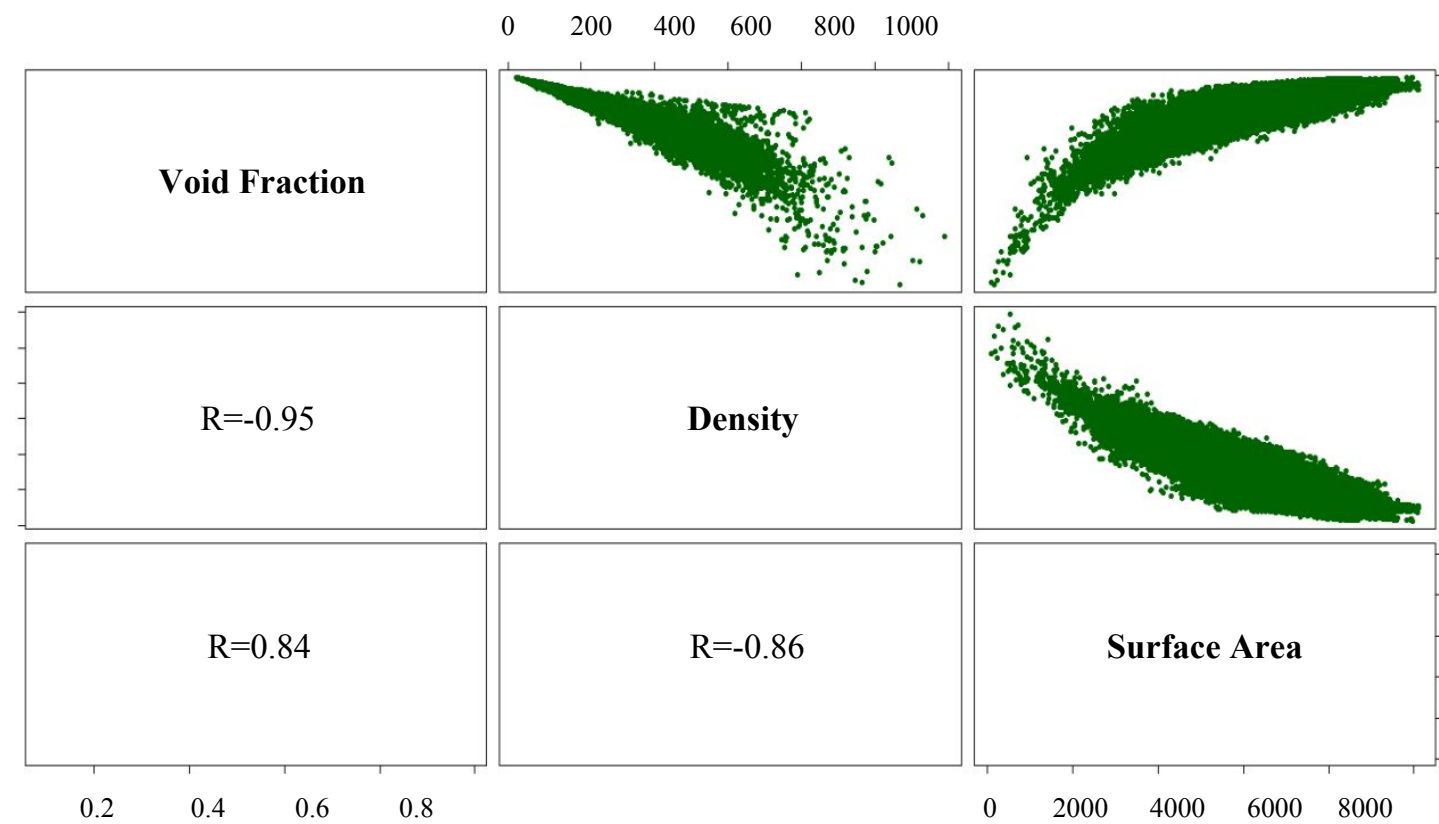

b

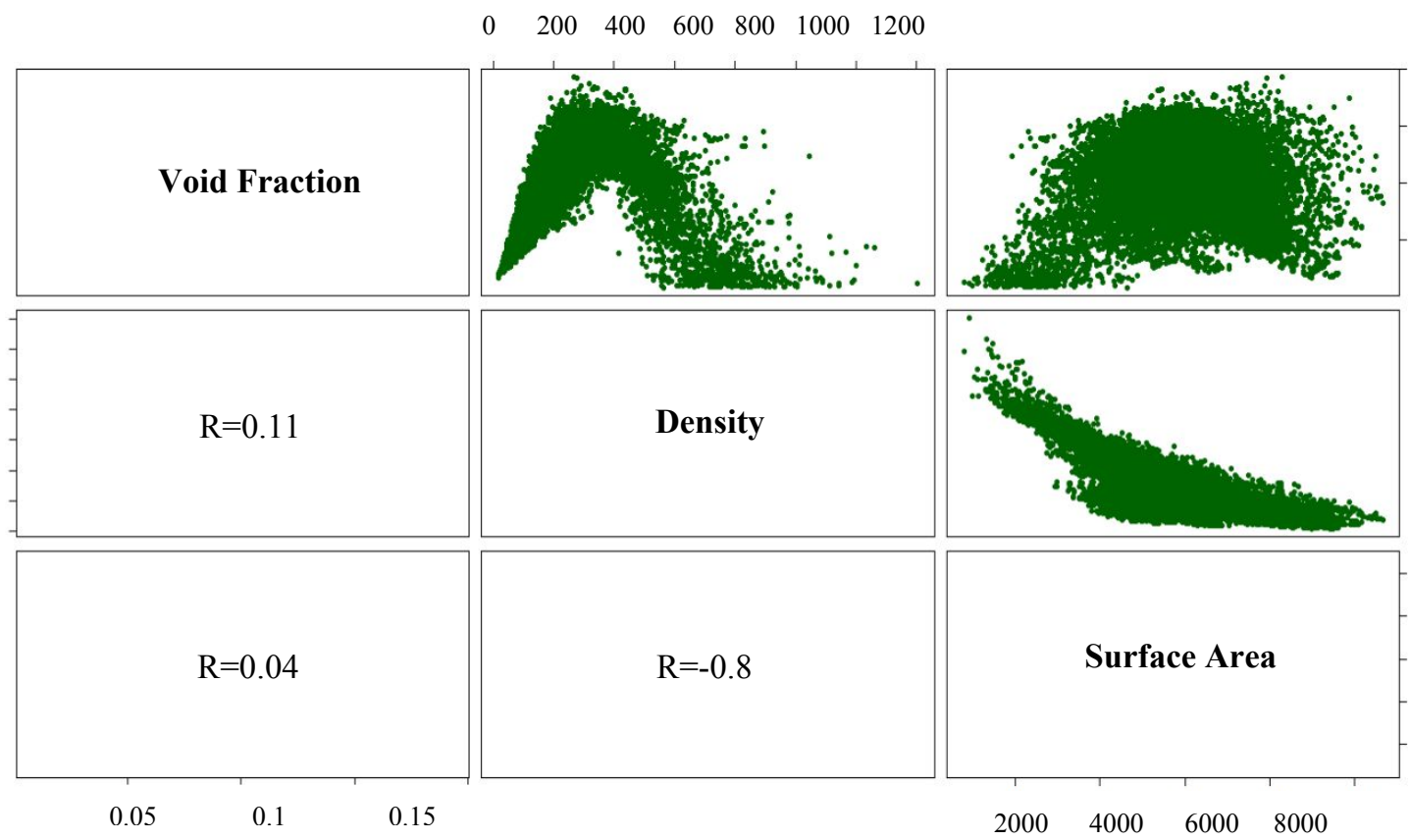

Figure S12. Pair plot of structural features for (a) COFs and (b) PPNs. 


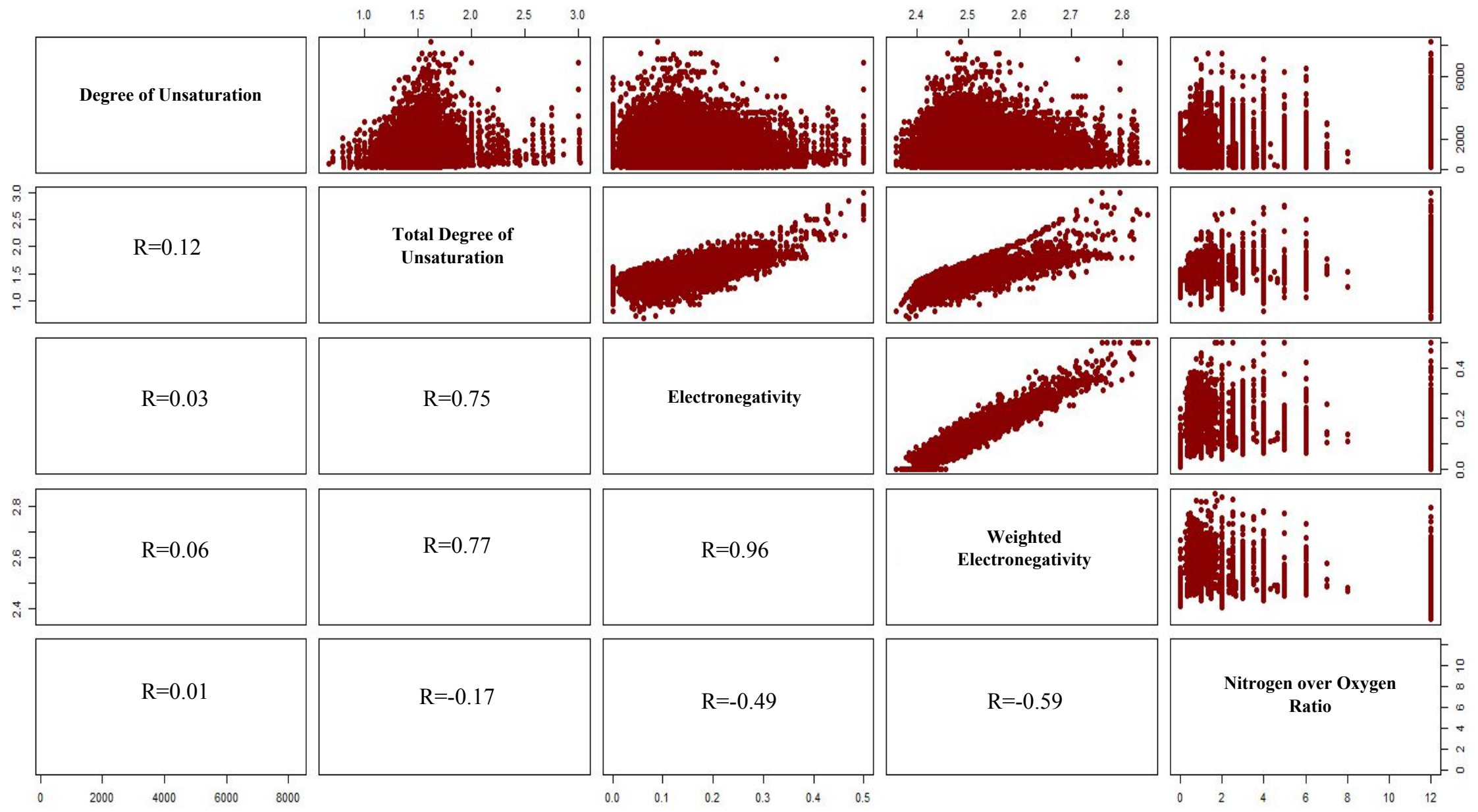

Figure S13. Pair plot of Chemical Features for COFs 


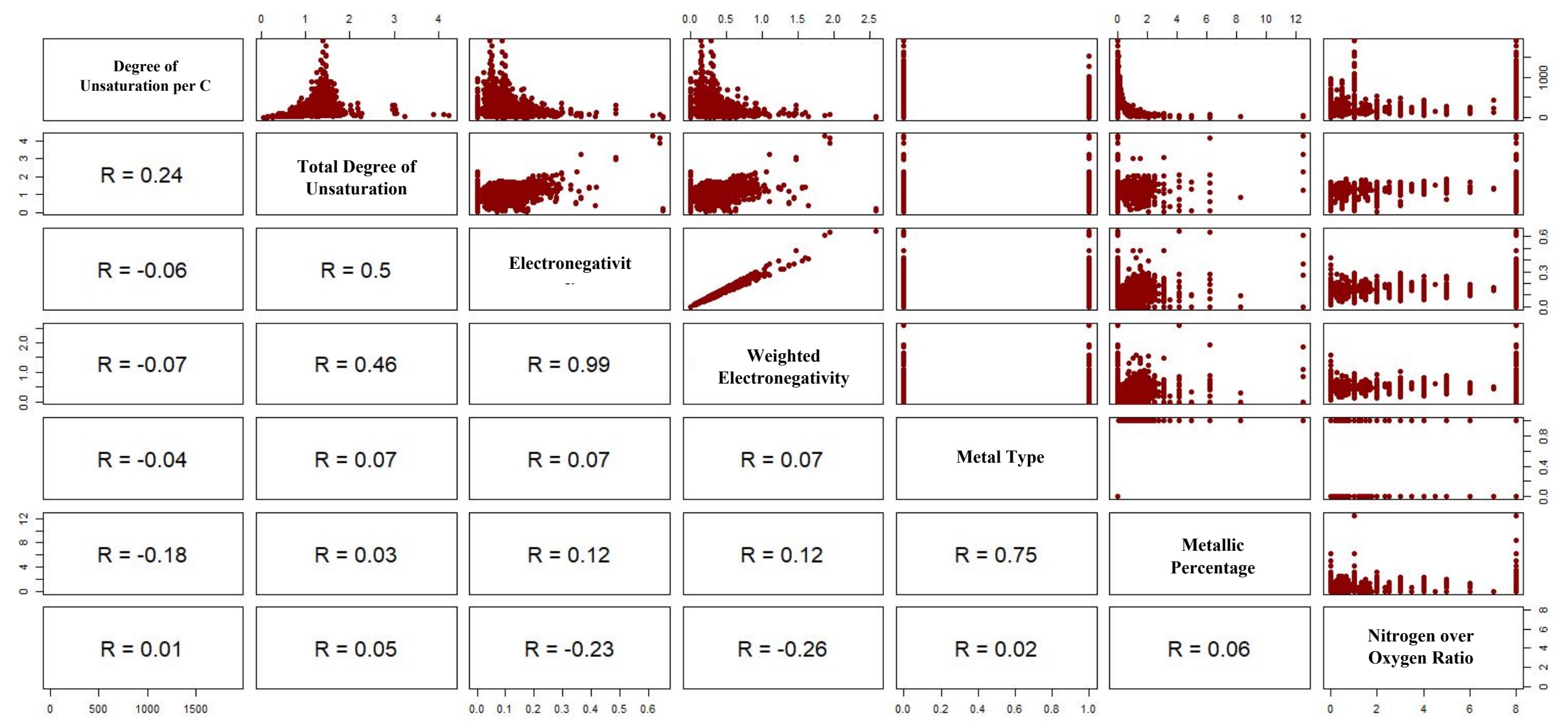

Figure S14. Pair plot of Chemical Features for PPNs. 
a)

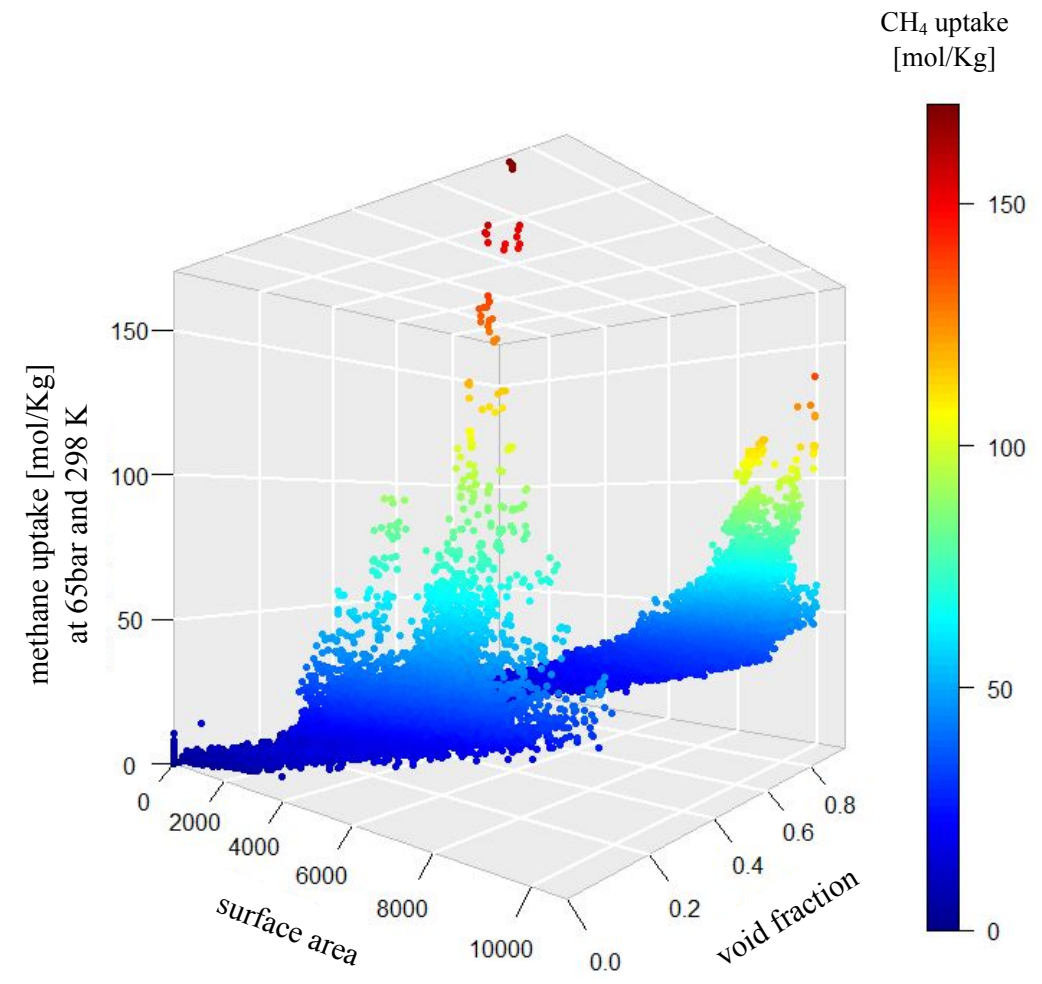

$\mathrm{CH}_{4}$ uptake $[\mathrm{mol} / \mathrm{Kg}]$

b)

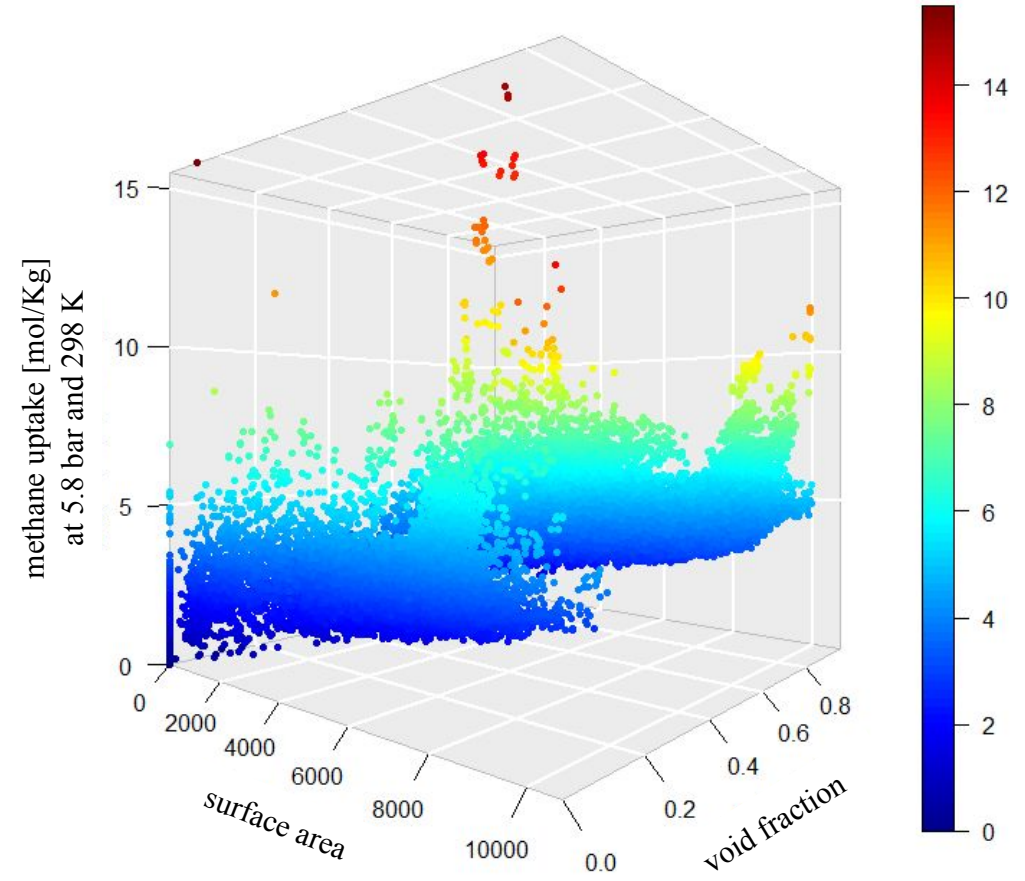

Figure S15. 3D presentation of methane adsorption $[\mathrm{mol} / \mathrm{Kg}]$ vs. surface area $\left[\mathrm{m}^{2} / \mathrm{g}\right]$ vs. void fraction at 65 and 5.8 bar respectively on combined COFs-PPNs data respectively. 
a)

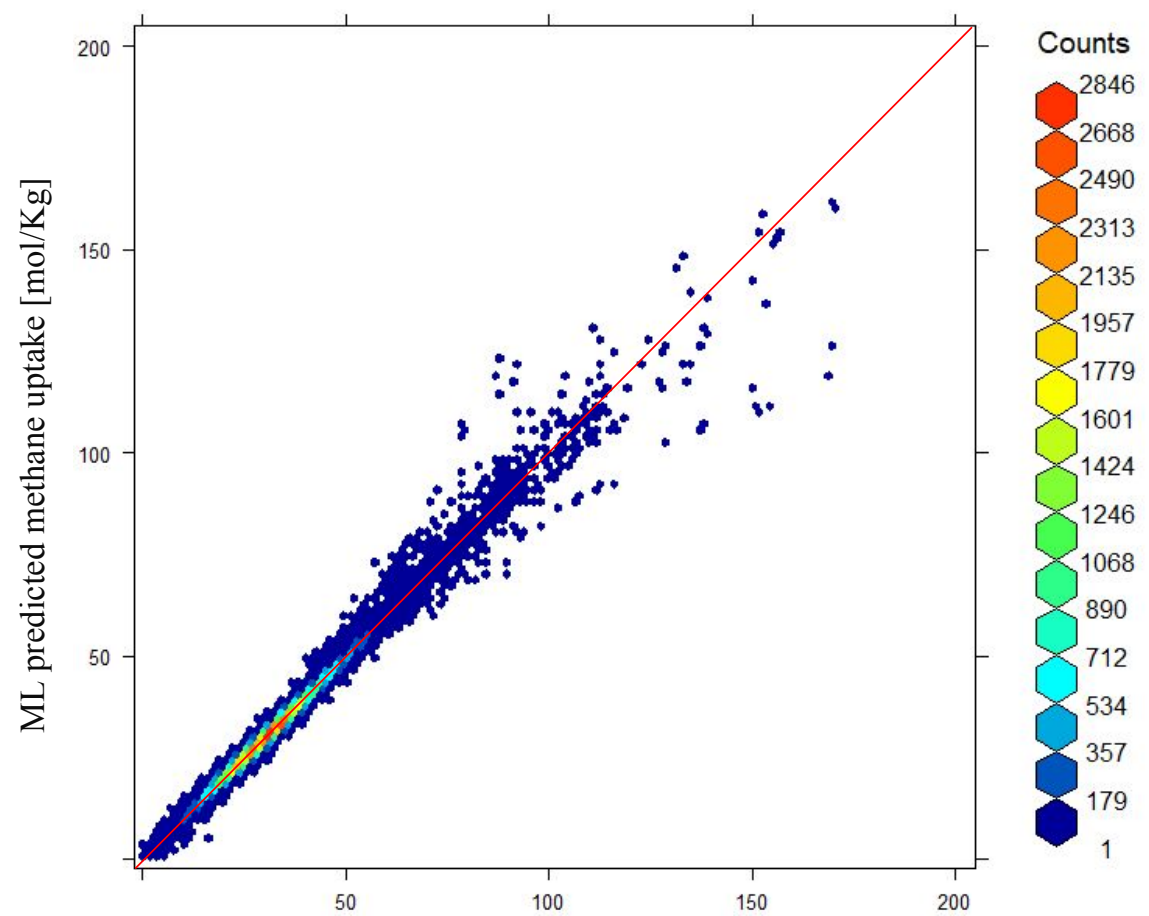

b)

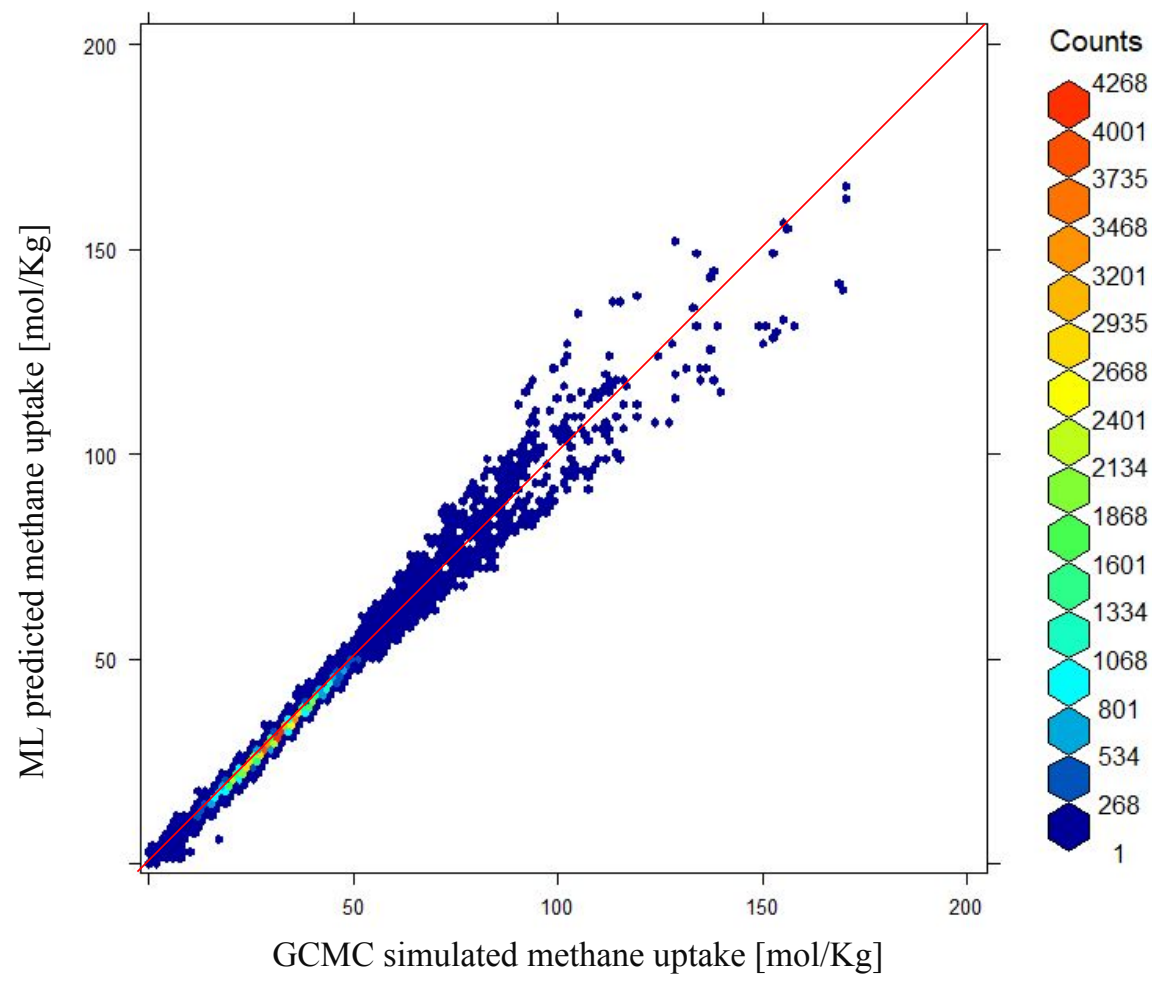

Figure S16. Parity plots for predicted (ML) vs. GCMC simulated methane uptake $[\mathrm{mol} / \mathrm{Kg}]$ at 65 bar and 298 K on COFs-PPNs data using (a) Structural only features, (b) combined structural and chemical features. 
a)

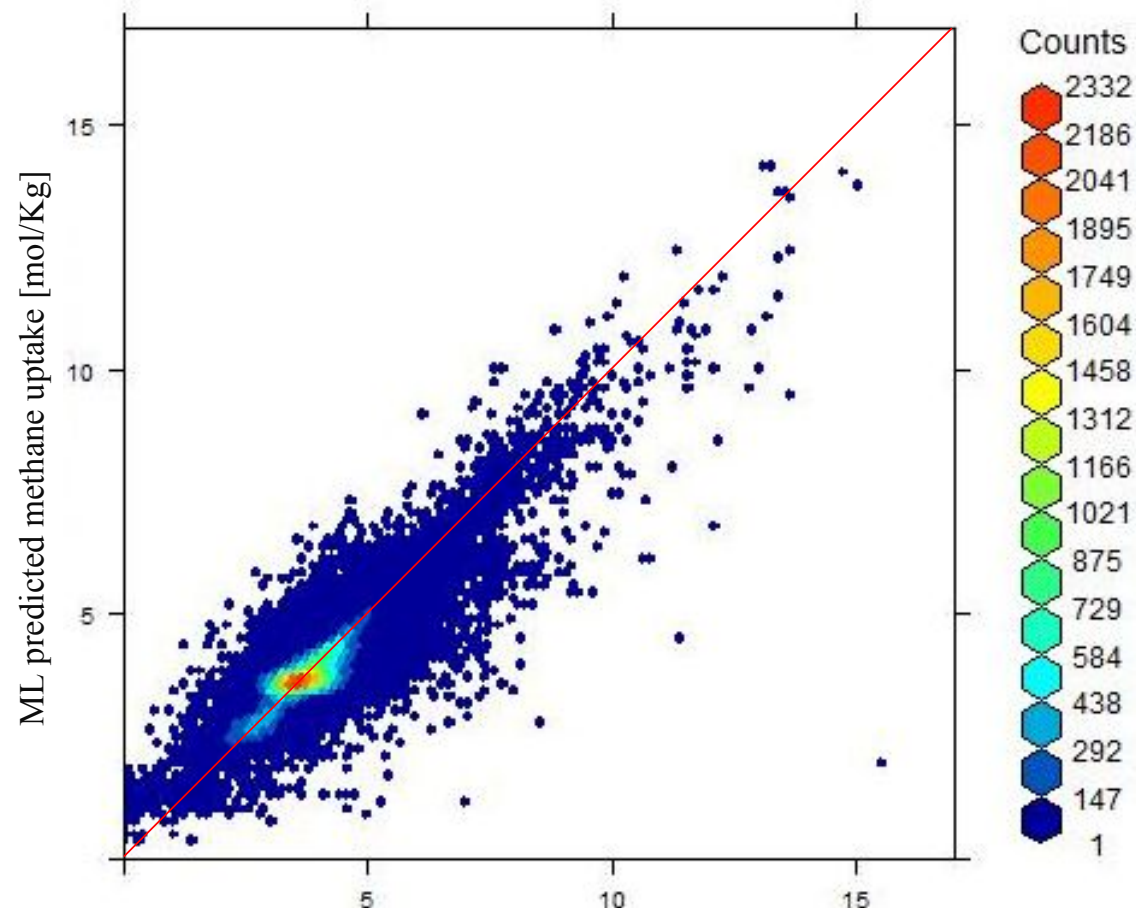

b)

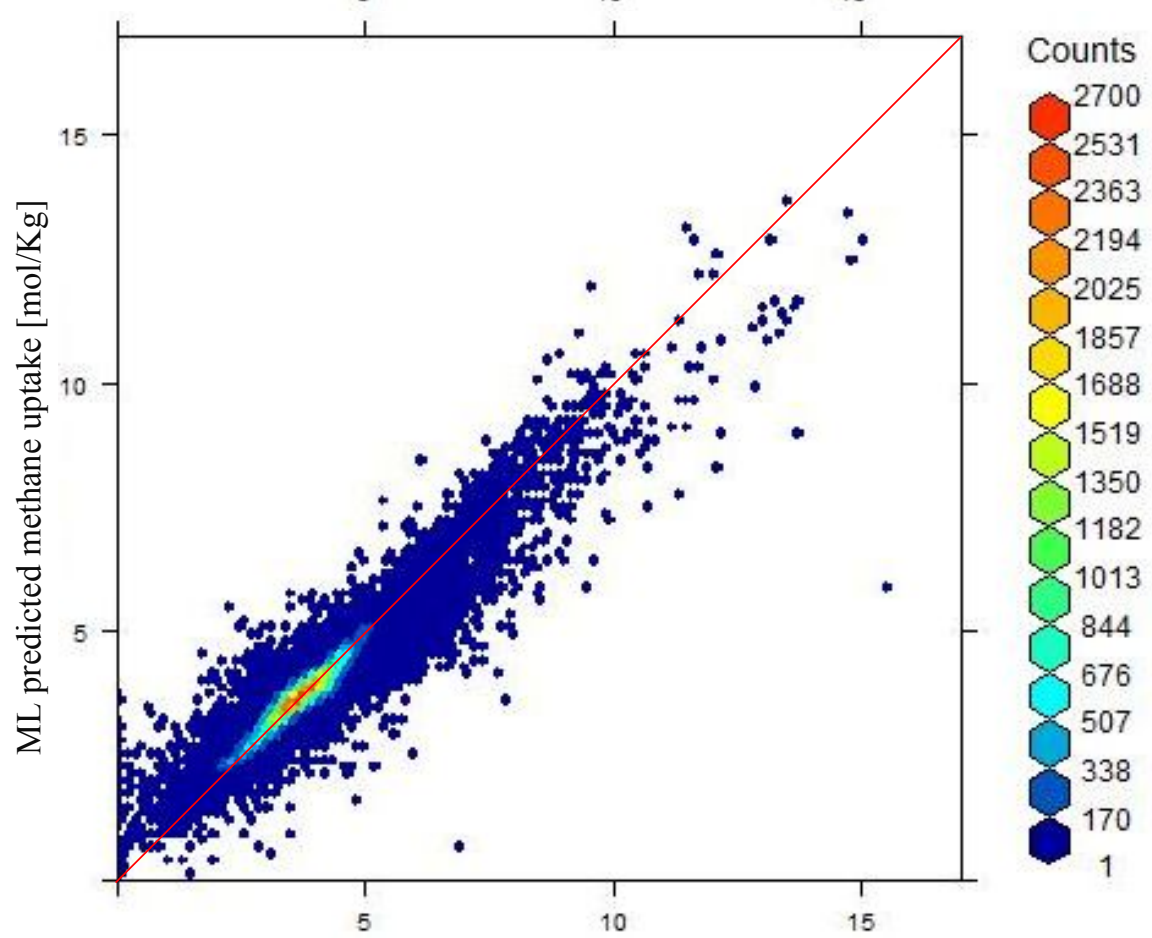

GCMC simulated methane uptake $[\mathrm{mol} / \mathrm{Kg}]$

Figure S17. Parity plots for predicted (ML) vs. GCMC simulated methane uptake $[\mathrm{mol} / \mathrm{Kg}]$ at 5.8 bar and 298 K on COFs-PPNs data using (a) Structural only features, (b) combined structural and chemical features. 
a)

density

void fraction

surface area

weighted electronegativity

total degree of unsaturation

electronegative atoms to total atoms

number of hydrogen

degree of unsaturation per carbon

number of carbon

number of fluorine

number of oxygen

number of nitrogen

number of chlorine

number of germanium

number of silicon

number of sulfur

number of iodine

number of phosphorus b)

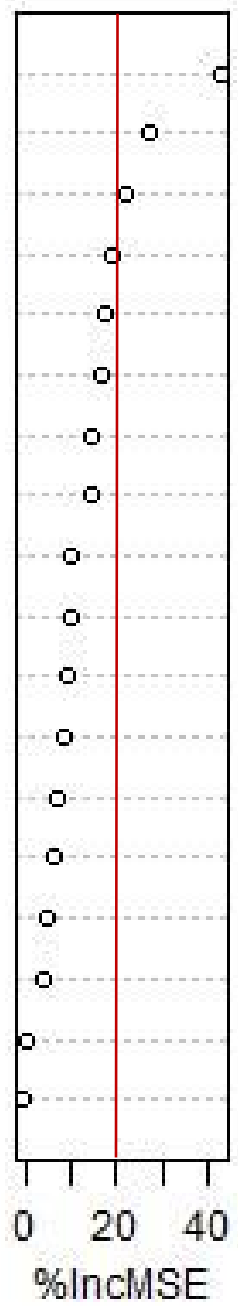

density

total degree of unsaturation

electronegative atoms to total atoms

surface area

number of oxygen

number of nitrogen

number of silicon

void fraction

weighted electronegativity

degree of unsaturation per carbon

number of hydrogen

number of fluorine

number of carbon

number of sulfur

number of germanium

number of chlorine

number of iodine

number of phosphorus

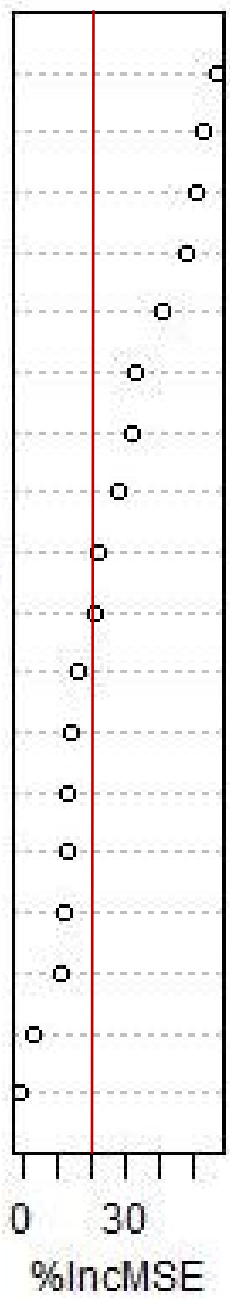

Figure S18. Feature importance (from random forest algorithm) for methane adsorption at (a) high pressure (65 bar) and low pressure (5.8 bar) on COFs-PPNs data. 